\section{A Occult Transverse Lacer- ation with Only Mesenter- ic Hematoma: No Free Air, No Ascitic Fluid, No Bowel Thickening in CT Do Not Give us a Guarantee in Which Surgically Important Bowel Injury is Absent}

\section{Tadao Kubota*, Ken Mizokami, Mayu Shimaguchi, Hideki Katagiri, Kenji Okumura, Kentaro Yoshikawa and Takashi Sakamoto}

Department of General Surgery, Tokyo Bay Medical Center, Todaijima, Urayasu city, Japan

\begin{abstract}
Background: In case of blunt abdominal trauma, the presence of free air, free ascitic fluid and bowel thickening are common findings of hollow viscous injury. Here in, we present a rare case of colonic perforation in which only mesenteric hematoma was observed.

Case presentation: 52 year old male was brought to our institute with upper abdominal pain. Several hours before he noticed his pain, he had fallen down on a road. On physical examination his abdomen was almost benign except for the slight tenderness on his epigastric area. Initial CT scan showed a hematoma like lesion localized in the transverse-mesentery. Because there were no free air, no ascitic fluid and no bowel thickening, he had been managed non-operatively. Three days after admission, he had high fever. The repeat CT showed extraluminal air. Emergent surgery was done. Intraoperative finding revealed major laceration of the transverse colon. Postoperative course was almost good.

Discussion and conclusion: Free air, intra-peritoneal ascitic fluid and bowel thickening in CT scan are potent evidences of bowel perforation, but none of these findings do not give us a guarantee the absence of surgically important bowel injury.
\end{abstract}

*Corresponding author: Tadao Kubota, Department of General Surgery, Tokyo Bay Medical Center, Todaijima, Urayasu city, Japan, Tel: +81 473513101; E-mail: tadaokubota@mac.com

Citation: Kubota T, Mizokami K, Shimaguchi M, Katagiri H, Okumura K, et al. (2016) A Occult Transverse Laceration with Only Mesenteric Hematoma: No Free Air, No Ascitic Fluid, No Bowel Thickening in CT Do Not Give us a Guarantee in Which Surgically Important Bowel Injury is Absent. J Emerg Med Trauma Surg Care 3: 15.

Received: December 16, 2015; Accepted: April 19, 2016; Published: April 30, 2016
Keywords: CT; Injury; Transverse colon

\section{Background}

Early detection of surgically important bowel and mesenteric injury is one of challenging problem for trauma surgeons. Atri et al., reported that sensitivity and specificity for initial evaluation of bowel and mesenteric injury are 87 to $95 \%$ and 48 to $84 \%$ respectively [1]. If there are no findings, rule-out is easy. But if there are some indirect sings, rule-in is relatively difficult. So we sometimes have missed diagnosis and delayed surgery cases. Extraluminal air is very potent findings, so it can lead to the definitive diagnosis of bowel perforation. Intraperitoneal ascitic fluid is common indirect findings. A locally isolated bowel thickening and ascitic fluid are the strong value of the presence of bowel perforation. When we can see none of these findings, we usually think that there may not be surgically important bowel injury. As far as we know, major bowel laceration without extraluminal air, ascitic fluid or bowel wall thickening is extremely rare.

\section{Case Presentation}

52 years old male was brought to our emergency department with sever upper abdominal pain. About five hours before he felt abdominal pain, he fell down on a road in the way home after drinking at a pub. He had not remembered whether he had been hit his stomach or not, because he had been heavily drunk. He only knew that he might fall on a road in his way home. There was no witness. Just after he came home, he didn't complained abdominal pain and fell asleep soon. A few hours later he got up because of severe upper abdominal pain. So his family took him our hospital. When he arrived at the emergency department, his upper abdominal pain had been continued without any other symptoms. His blood pressure was $116 / 65 \mathrm{mmHg}$, heart rate was $70 / \mathrm{min}$, respiratory rate was $18 / \mathrm{min}$, temperature was $37.0^{\circ}$, GCS was 15 points. On the inspection, there were no bruises or abrasions on his surface. On the physical examination, his head, neck, chest, pelvis, extremities, and back were normal. His abdomen was overall soft and no muscle defense, but there was a localized tenderness on the epigastric lesion without rebound tenderness. Bowel sound was normal activity. The emergency physician thought that he might have abdominal trauma, so enhanced CT scan was ordered. The initial CT revealed a low density area located into the transverse mesocolon which was thought as hematoma without extravasation (Figure 1). There were no extra-luminal air, no ascitic fluid and no bowel thickening. The emergency physician consulted him to trauma surgeons. There was a big question that he had been injured or not. We had two hypotheses. One was mesenteric injury; the other was spontaneous mesenteric hemorrhage. If he had been injured, both the physical examination and image study didn't support the presence of bowel perforation. In case of the spontaneous bleeding, there was no indication of hemostasis because of the vital sign was stable and no extravasation was observed in enhanced CT. We thought both situations could be managed non-operatively, so emergency surgery was withheld. Bowel rest and IV fluid therapy were started. At that 
Citation: Kubota T, Mizokami K, Shimaguchi M, Katagiri H, Okumura K, et al. (2016) A Occult Transverse Laceration with Only Mesenteric Hematoma: No Free Air, No Ascitic Fluid, No Bowel Thickening in CT Do Not Give us a Guarantee in Which Surgically Important Bowel Injury is Absent. J Emerg Med Trauma Surg Care 3: 15.

time, we thought he had not injured as followed reasons. He clearly remembered the onset of his abdominal pain which was several hours after the event. If he fell down on a road, no skin injury was unusual. We know the early diagnosis of hollow viscus injury is sometimes difficult; no free air is insufficient evidence of no bowel injury. However we never seen a case of bowel laceration without any ascitic fluid in CT scan. He had been stable for a couple of days. He had not complained any abdominal pain except for slight tenderness. In the morning on day three, he started to take a meal. In that afternoon his temperature had elevated over $38^{\circ}$ without abdominal pain. Second CT was done and it revealed some extra-luminal air around the transverse-colon which was definitive evidence of bowel perforation (Figure 2). The day before, we had found some subcutaneous hematoma on his face, right shoulder and right thigh. After all, we changed our mind he must have been injured by some external forces at that night. Immediately, the patient went to operating room for emergency surgery. When we open the abdomen, we found a localized hematoma into the mid transverse colon. The serosa of the transverse mesocolon was completely intact. There were not fecal contaminations in the peritoneal cavity. The involved transverse colon was resected and primary anastomosis was done. There were some small hematomas in the omentum but no other organ injury was seen. Before finishing the operation, we made a temporary diverting loop ileostomy for protection of colon anastomosis. Because, intramesenteric space was contaminated by the feces. In the resected transverse colon, a major laceration was observed which was just located on the mesenteric side (Figure 3). The pathological study revealed no remarkable reasons of spontaneous perforation such as diverticulitis, inflammation, or tumor. The postoperative course was almost good and the temporary ileostomy was closed four weeks after initial surgery.

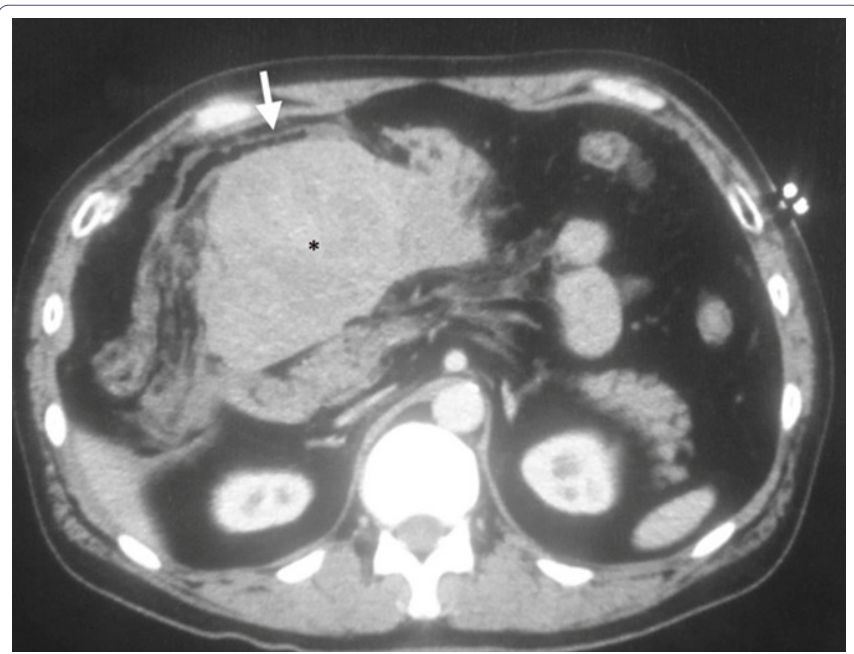

Figure 1: Initial CT.

We can see a transverse mesenteric hematoma $\left(^{*}\right)$. Around there, no extraluminal air and no free fluid are observed. No wall thickening is seen in connected transverse colon (arrow).

Actually we had thought his abdominal pain was not related to trauma at the time of admission. In initial CT, hematoma like fluid collection is packed into the transverse mesocolon which was only finding. It thought to be rare, if it was related to blunt abdominal trauma. We couldn't get any clear evidence he had blunt abdominal trauma. Before and after surgery, we asked him several times whether he remembers or not how he fell down and hit him stomach. However his answer had not been changed. Every time he said he did not

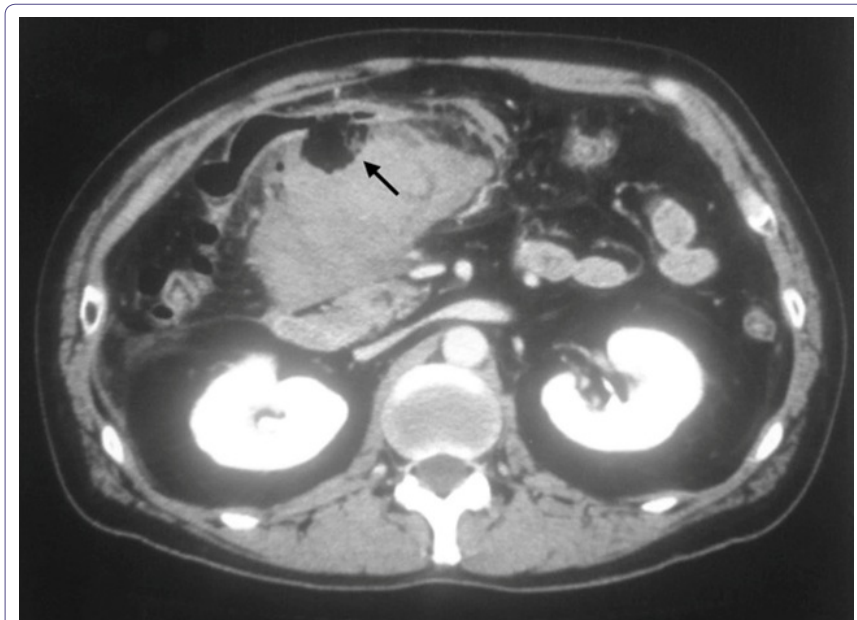

Figure 2: Repeat CT (3 days after).

Arrow shows extraluminal air in the mesenteric hematoma. Free air and free fluid are still not seen there.

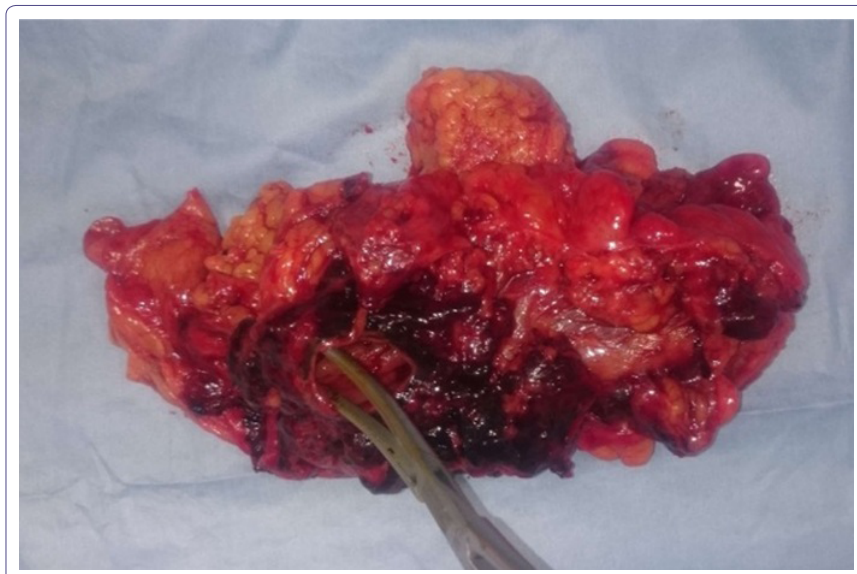

Figure 3: Resected specimen.

The forceps shows a major laceration of transverse colon which is located in mesenteric side.

remember at all except for only the fact he fell. Given that he had been had some subcutaneous hematoma and the intraoperative view, he must have blunt trauma. The reason why he didn't know the fact of his abdominal trauma is thought that he was deeply drunk. It remains still a suspicion. His subcutaneous hematoma were multiple and apart from each other. The face bruise was on his left cheek, right shoulder one was on the front side, right thigh one was on the back. It is difficult to explain all injuries caused by one time hit. If he really fell on a road, no abrasions and no lacerations on his surface were unusual. We have suspected that he might be received violence by somebody, but we have no idea to know the truth.

\section{Discussion}

In case of blunt abdominal trauma, free air in the peritoneum seen on CT scan is highly suggestive findings of bowel perforation. But its sensitivity is not so high, it is said that it seen in only $20-55 \%$ of patients [2,3]. Intraperitoneal free ascitic fluid is one of most sensitive indirect features of bowel and mesenteric injury. It is reported to occur in $93 \%$ of patients of all bowel and/or mesenteric injury [2]. The presence of a moderate to large amount of ascitic fluid is a strong indicator for exploration. On the other hand, the absence of ascitic fluid is usually high negative predictive value in the exclusion 
of surgically important bowel injury [4]. The laceration of transverse colon with mesenteric injury is common in blunt abdominal trauma. When it occurs on the mesenteric side, connected mesentery is usually disrupted. We had never seen a case of major laceration of the transverse colon without free perforation in blunt trauma. If the transverse colon and mesentery was hit by strong force directly, the serosa would not be intact. If it was intra-luminal baro-trauma, it would be extra-luminal air. Isolated, localized bowel wall thickening is also associated with high likelihood of bowel injury that requires surgically repair [3]. Large bowel wall thickening had a high specificity of $97 \%$ in detecting bowel injury [1]. In this case, there was no bowel thickening in the transverse colon not only in initial CT but also in repeat CT. Major laceration with only mesenteric hematoma is rare. As far as we examined for available medical search system, we couldn't find any same cases. Meissenizer, et al., reported two cases of mesenteric hematoma with occult small intestinal injury [5]. Through this rare case, we learned that when we find prominent findings in a $\mathrm{CT}$, non operative management is not safe even if there are no typical findings of bowel perforation. Although non-traumatic spontaneous mesenteric or retroperitoneal hemorrhage is reported in some literatures [6-8], anticoagulation therapy such as warfarin is administrated in all cases. Our patient had not been taken any anticoagulations.

\section{Conclusion}

As a result, we retrospectively thought there were no clear indications of immediate laparotomy in initial stage. More than anything his abdomen was benign and no peritoneal sings. However we re-learned the fact delayed presentation of major bowel injury is not rare in blunt trauma. Emphasis should be based on having a suspicious and close observation is mandatory.

\section{References}

1. Atri M, Hanson JM, Grinblat L, Brofman N, Chughtai T, et al. (2008) Surgically important bowel and/or mesenteric injury in blunt trauma: accuracy of multidetector CT for evaluation. Radiology 249: 524-533.

2. Brofman N, Atri M, Hanson JM, Grinblat L, Chughtai T, et al. (2006) Evaluation of bowel and mesenteric blunt trauma with multidetector CT. Radiographics 26: 1119-1131.

3. Strouse PJ, Close BJ, Marshall KW, Cywes R (1999) CT of bowel and mesenteric trauma in children. Radiographics 19: 1237-1250.

4. Levine CD, Patel UJ, Wachsberg RH, Simmons MZ, Baker SR, et al. (1995) $\mathrm{CT}$ in patients with blunt abdominal trauma: clinical significance of intraperitoneal fluid detected on a scan with otherwise normal findings. AJR Am J Roentgenol 164: 1381-1385.

5. Meissnizer MW, Stättner S, Meissnizer T (2014) Small mesenteric hematoma following blunt abdominal trauma as early sign in computed tomography of occult small bowel perforation-report of 2 cases. Emerge Radiol 21: 647-650.

6. Hosseini H, Hosseinzadeh A, Rauflan K, Hedjazi A (2015) Nontraumatic Retroperitoneal Hematoma after Warfarin Administraton: Fetal Case Report and Review of the literature. Am J Forensic Med Pathol 36: 245-248.

7. Lissoway J, Booth A (2010) Fatal retroperitoneal hematoma after enoxaparin administration in a patient with paroxysmal atrial flutter. Am J Health Syst Pharm 67: 806-809.

8. Won DY, Kim SD, Park SC, Moon IS, Kim JI (2011) Abdominal compartment syndrome due to spontaneous retroperitoneal hemorrhage in a patient undergoing anticoagulation. Yonsei Med J 52: 358-361. 